\title{
Spin Domains in One-Dimensional Conservative Polariton Solitons
}

Maksym Sich, ${ }^{\dagger}$ Lucy E. Tapia-Rodriguez, ${ }^{\dagger}$ Helgi Sigurdsson, ${ }^{\ddagger}$ (†) Paul M. Walker, ${ }^{\dagger}$ Edmund Clarke, ${ }^{\mathbb{I}}$ Ivan A. Shelykh, ${ }^{\ddagger}, \S$ Benjamin Royall, ${ }^{\dagger, \|}$ Evgeny S. Sedov, ${ }^{\perp, \#}$ Alexey V. Kavokin, ${ }^{\perp,} \square, \triangle$ Dmitry V. Skryabin, ${ }^{\S, \nabla}$ Maurice S. Skolnick, ${ }^{\dagger, \S}$ and Dmitry N. Krizhanovskii ${ }^{*}, \dagger, \S$

${ }^{\dagger}$ Department of Physics and Astronomy, The University of Sheffield, Sheffield, S3 7RH, United Kingdom

${ }^{\ddagger}$ Science Institute, University of Iceland, Dunhagi-3, IS-107 Reykjavik, Iceland

"EPSRC National Epitaxy Facility, The University of Sheffield, Sheffield, S1 4DE, United Kingdom

${ }^{\S}$ Department of Nanophotonics and Metamaterials, ITMO University, St. Petersburg, 197101, Russia

"Huawei, B55 Adastral Park, Ipswich, IP5 3RE, United Kingdom

${ }^{\perp}$ School of Physics and Astronomy, University of Southampton, Southampton, SO17 1NJ, United Kingdom

\#Vladimir State University, Gorky Street 87, Vladimir, 600000, Russia

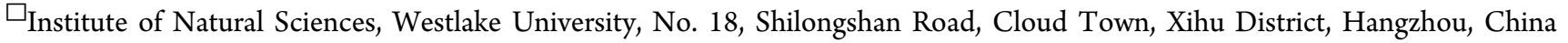

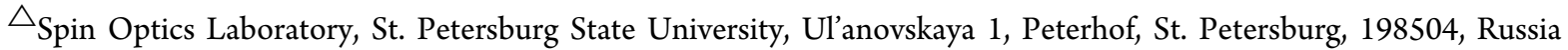

$\nabla_{\text {Department of Physics, University of Bath, Bath, BA2 7AY, United Kingdom }}$

\section{Supporting Information}

ABSTRACT: We report stable orthogonally polarized domains in highdensity polariton solitons propagating in a semiconductor microcavity wire. This effect arises from spin-dependent polariton-polariton interactions and pump-induced imbalance of polariton spin populations. The interactions result in an effective magnetic field acting on polariton spin across the soliton profile, leading to the formation of polarization domains. Our experimental findings are in excellent agreement with theoretical modeling taking into account these effects.
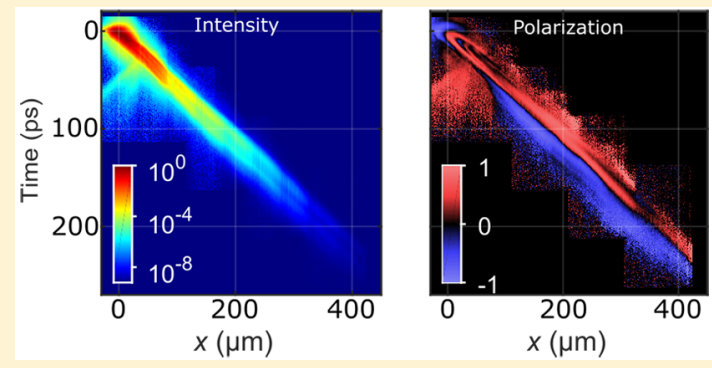

KEYWORDS: microcavity, exciton-polariton, soliton, one-dimensional kinetics, nonlinear physics, TE-TM splitting.

$\mathrm{T}$ emporal and/or spatial domains of coupled multiple dipoles play a significant role in the properties of various physical systems. Magnetic domains in (anti)ferromagnets have been widely studied to date and are utilized in modern memory devices (hard drives). Domain formation with electric dipoles has also been observed in atomic spinor Bose-Einstein condensates. ${ }^{1}$ In optics, polarization domain formation is governed by modulation instability with the resultant separation of adjacent domains by a domain wall, a topological defect closely linked to soliton formation. ${ }^{2-4}$ While scalar nonlinear effects related to solitons ${ }^{5,6}$ (or optical supercontinuum ${ }^{7}$ ) have been studied extensively, little attention has been given to the spatiotemporal evolution of the polarization (or spin) degree of freedom in vectorial optical structures. Only recently have timelocalized polarization rotations or polarization domain walls been reported for light traveling in nonlinear optical fibers ${ }^{8}$ and for dissipative solitons in vertical-cavity surface-emitting lasers. ${ }^{9}$

In this paper we study spatiotemporal polarization domains in a system of exciton-polaritons in semiconductor microcavities. In such highly nonlinear systems observation of a variety of quantum fluid phenomena, ${ }^{10,11}$ Bose-Einstein condensation, ${ }^{12}$
Berezinskii-Kostelitz-Thouless phases, superfluidity, and dark and bright solitons ${ }^{13}$ have all been reported.

Polaritons are characterized by two possible spin projections on the structure growth axis, which correspond to two opposite circular polarizations. ${ }^{14}$ Efficient external control of the polariton spin makes microcavity-based structures promising building blocks for spin-optronic devices, i.e., optical equivalents of spintronic devices. ${ }^{15}$ Moreover, due to the exchange terms that dominate the exciton-exciton scattering, ${ }^{16}$ polaritonpolariton interactions are strongly spin anisotropic: polaritons with the same spin projections strongly repel each other, while polaritons with parallel spins interact more weakly and sometimes even attract each other. ${ }^{17,18}$ The interplay between spin dynamics and polariton-polariton interactions leads to a wide variety of nonlinear polarization phenomena in microcavities. This includes spin switching, ${ }^{19}$ spin-selective filtering, ${ }^{20}$ polarization-dependent stability of dissipative solitons, ${ }^{21}$ optical analogues of magnetically ordered states, ${ }^{22,23}$ dark half solitons, ${ }^{24}$ and spin and half vortices. ${ }^{25,26}$

Received: October 9, 2018

Published: November 12, 2018 
Here we demonstrate formation of polariton spin domains within high-density wavepackets evolving into conservative soliton(s) in a quasi-1D system. Experimentally we resolve the full Stokes polarization vector of the propagating wavepackets under a wide range of excitation powers and develop a theoretical model that we validate through numerical simulations, qualitatively reproducing the experimental results. The experiments were performed in a microcavity wire (MCW): 5 $\mu \mathrm{m}$ wide and $1 \mathrm{~mm}$ long mesas etched from planar microcavities. Thanks to the 1D nature of polariton wires and the long polariton lifetimes $(\sim 30 \mathrm{ps})$, we could reach polariton densities sufficient for formation of solitons. ${ }^{27}$ At low excitation powers we observe polarization precession caused by a linear in-plane effective magnetic field inside the sample. At higher excitation powers the high polariton density and an imbalance of polariton spin populations lead to an extra out-of-plane magnetic field. This nonlinear field causes, first, fast polarization oscillations and then the formation of spin domains.

\section{RESULTS}

We performed our experiments on a $3 \lambda / 2$ microcavity composed of three embedded InGaAs quantum wells $(10 \mathrm{~nm}$ thick, 4\% indium) and GaAs/AlGaAs ( $85 \% \mathrm{Al})$ distributed Bragg mirrors with 26 (23) repeats on the bottom (top) mirror. The Rabi splitting and the polariton lifetime are $\sim 4.12 \mathrm{meV}$ and $\sim 30$ ps in this sample, which was previously described in refs 27 and 28. The detuning between the exciton and the photon modes is ca. $-2 \mathrm{meV}$. The top mirror was partially etched (down to the last few layers of the top distributed Bragg reflection (DBR)) defining $1000 \mu \mathrm{m}$ long mesas (MCWs) of different widths. In all our measurements we used the same $5 \mu \mathrm{m}$ wide MCW as in ref 27.

We excited the sample using a TE-polarized pulsed $(\sim 5$ ps full width at half-maxima, fwhm) laser quasi-resonant with the lower polariton branch corresponding to the ground MCW mode. The angle of incidence was $k_{x} \simeq 2.2 \mu \mathrm{m}^{-1}$, at which the effective polariton mass is negative, favoring formation of solitons. ${ }^{27} \mathrm{We}$ employed transmission geometry, where we applied the excitation beam on one side of the cavity (bottom) and collected emission on the opposite side (top) to avoid the reflected pump beam saturating our detectors. We then changed the laser power and measured the full Stokes vector of the emitted light as a function of time and propagation coordinate, $x$, for each excitation power. In the experiment we separately detected emission intensity in six polarizations: horizontal $\left(I_{\mathrm{h}}\right)$, vertical $\left(I_{\mathrm{v}}\right)$, diagonal $\left(I_{\mathrm{d}}\right)$, antidiagonal $\left(I_{\mathrm{ad}}\right)$, and right-hand $\left(I_{\sigma^{+}}\right)$and left-hand $\left(I_{\sigma^{-}}\right)$circularly polarized. In the circular polarization basis of the cavity light $\left\{\psi_{+} ; \psi_{-}\right\}$, the total intensity is written $S_{0}=\left|\psi_{+}\right|^{2}+\left|\psi_{-}\right|^{2}$ and the Stokes vector (normalized to unity) $S^{*}=\left(S_{1}^{*}, S_{2}^{*}, S_{3}^{*}\right)$ is given by

$$
\begin{gathered}
S_{1}^{*}=\frac{\psi_{+} \psi_{-}^{*}+\psi_{+}^{*} \psi_{-}}{\left|\psi_{+}\right|^{2}+\left|\psi_{-}\right|^{2}}=\frac{I_{\mathrm{h}}-I_{\mathrm{v}}}{I_{\mathrm{h}}+I_{\mathrm{v}}} \\
S_{2}^{*}=i \frac{\psi_{+} \psi_{-}^{*}-\psi_{+}^{*} \psi_{-}}{\left|\psi_{+}\right|^{2}+\left|\psi_{-}\right|^{2}}=\frac{I_{\mathrm{d}}-I_{\mathrm{ad}}}{I_{\mathrm{d}}+I_{\mathrm{ad}}} \\
S_{3}^{*}=\frac{\left|\psi_{+}\right|^{2}-\left|\psi_{-}\right|^{2}}{\left|\psi_{+}\right|^{2}+\left|\psi_{-}\right|^{2}}=\frac{I_{\sigma^{+}}-I_{\sigma^{-}}}{I_{\sigma^{+}}+I_{\sigma^{-}}}
\end{gathered}
$$

At the lowest excitation power, $P=13 \mu \mathrm{W}$, polariton nonlinearity is very weak and the wavepacket propagates in the

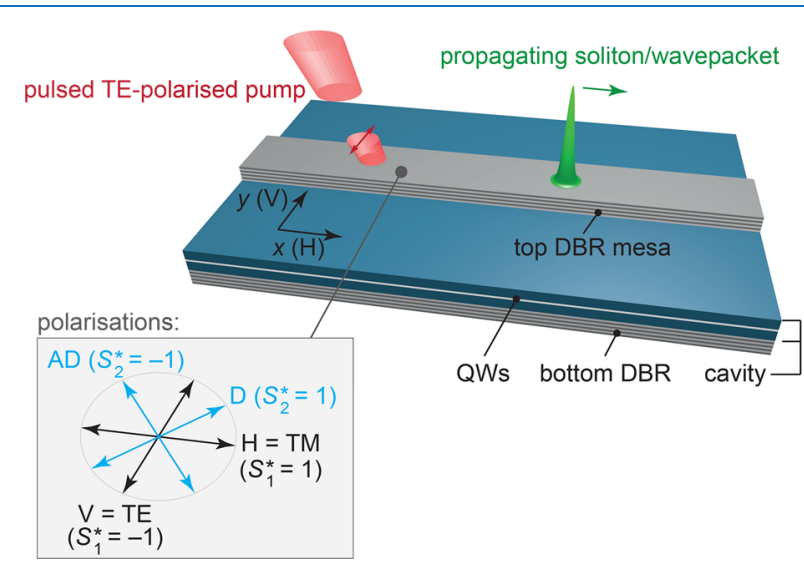

Figure 1. Scheme of the experiment. The partially etched top DBR mesa aligned along the longitudinal axis, $x$. Bottom-left inset shows linear polarizations mapped onto the laboratory frame relative to the sample position: horizontal $(\mathrm{H})$, vertical $(\mathrm{V})$, diagonal $(\mathrm{D})$, and antidiagonal $(\mathrm{AD})$ and their corresponding Stokes vector $\left(\mathrm{S}^{*}\right)$ component values.

linear regime, experiencing dispersive spreading (Figure 4 in the SI). At higher power, $P=87 \mu \mathrm{W}$, a soliton is formed, characterized by nonspreading propagation ${ }^{27,29}$ as shown in Figure 2a. Both circular and linear polarization degrees experience oscillations between negative and positive values with time as shown in the $S_{1}^{*}$ and $S_{3}^{*}$ components (Figure 2b,c) and the $S_{2}^{*}$ component (Figure 3 of the SI). We note that even though the pump beam is TE-polarized, the polarization of the emission at time $t=0$ has some diagonal and circular components, likely due to birefringence in the substrate and the influence of the edges of the MC wire on the polarization of the pump field before it couples to the polariton field inside the wire.

The polarization beats observed in Figure $2 b, c$ correspond to motion of the Stokes vector $\mathbf{S}^{*}$ (which is also referred to as the polariton pseudospin or spin) around the unit Poincare sphere, as shown in Figure 2d. where we plot the trajectory that the tip of the normalized Stokes vector follows as the soliton propagates. The direction of the Stokes vector is constructed by measuring the values of the Stokes components at different times at the spatial points of the soliton profile shown by the green lines in Figure $2 \mathrm{a}-\mathrm{c}$. The Stokes vector clearly precesses around the sphere as the soliton propagates. We note that at the lowest excitation power $(P=13 \mu \mathrm{W})$ the polariton wavepacket does not propagate long distances due to fast spreading, and the onset of the polarization beats and Stokes vector precession are barely visible (see Figure 3 of the SI).

Such motion of the Stokes vector around the Poincare sphere can be described mathematically as a precession of the polariton pseudospin around a time-varying effective magnetic field. ${ }^{30}$ Physically, this effective magnetic field arises from three mechanisms: TE-TM (V-H in the laboratory frame) splitting of polaritons propagating with nonzero momenta; ${ }^{24,31}$ strain, electronic anisotropy, or anisotropy related to crystallographic lattices $^{32,33}$ inducing splitting between $\mathrm{D}$ and $\mathrm{AD}$ polarized components; and polariton-polariton interactions. The last mechanism is weak at small powers $(P=87 \mu \mathrm{W})$, but starts playing a critical role at higher polariton densities $(P>0.95$ $\mathrm{mW})$, as we discuss below in the modeling section. The 

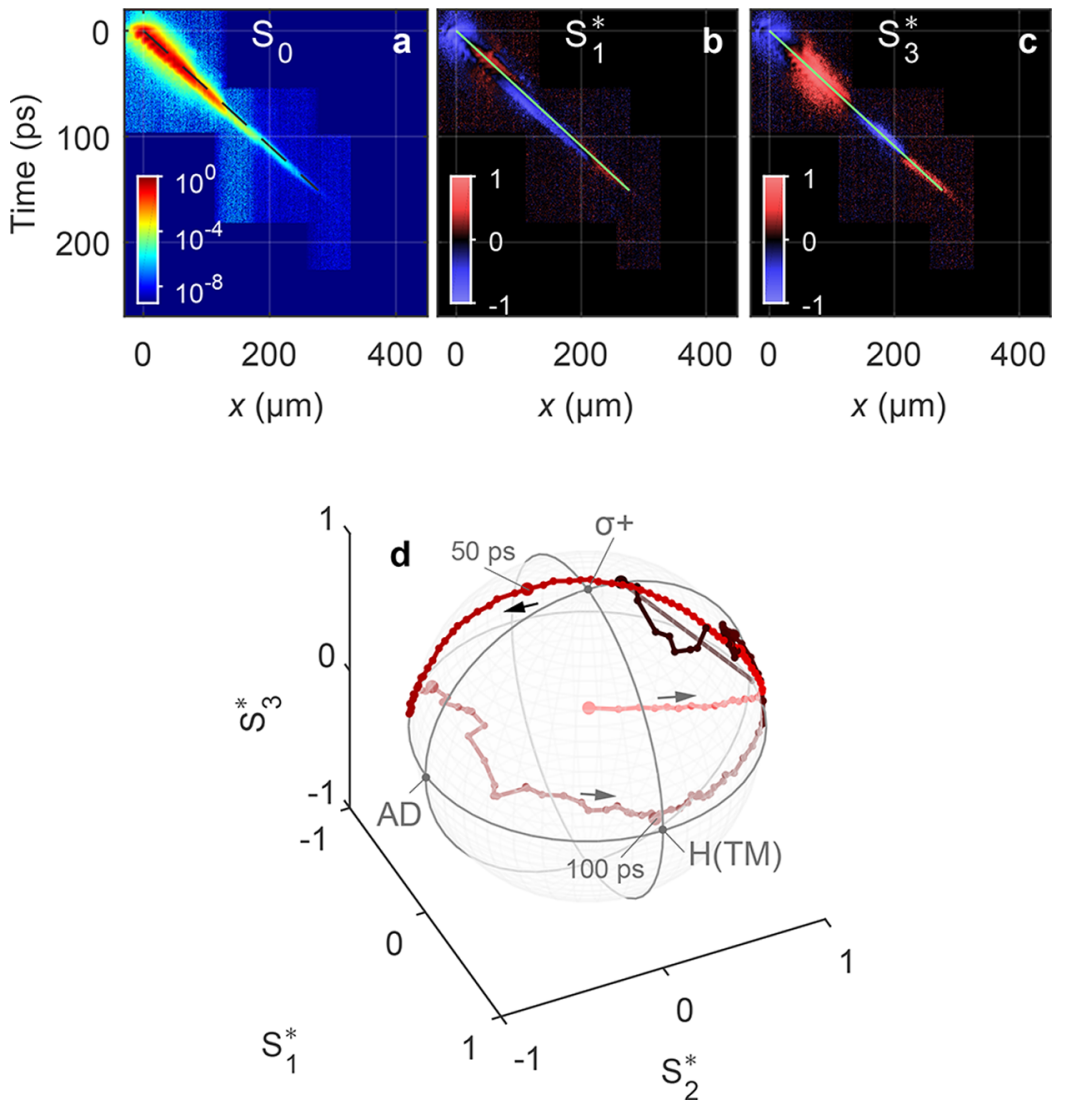

Figure 2. $P=87 \mu \mathrm{W}$. (a) Total emission intensity ( $S_{0}$, Stokes component) of a soliton as a function of coordinate $x$ and time. (b, c) Degree of polarization of the emission in linear $\left(S_{1}^{*}\right)$ and circular $\left(S_{3}^{*}\right)$ polarization bases. (d) Experimental evolution of the tip of the polariton Stokes vector on the surface of the Poincare sphere as a function of time. The length of the experimental Stokes vector is normalized to unity. The dimmer (brighter) traces correspond to the evolution on the Stokes vector on the back (front) surface of the sphere. The north and the south poles of sphere correspond to $\sigma^{+}$and $\sigma^{-}$circular polarizations, whereas the points on the equator correspond to the linear polarizations. The direction of the Stokes vector is constructed by measuring the values of the Stokes components at different times at the spatial points of the soliton profile shown by the green lines in panels $\mathrm{b}$ and $\mathrm{c}$. Data in $\mathrm{a}-\mathrm{c}$ are taken at $y=0 \pm 0.5 \mu \mathrm{m}$.

observed precession period of $T \simeq 60$ ps at $P=87 \mu \mathrm{W}$ (Figure $2 \mathrm{~b}-\mathrm{d})$ corresponds to approximately $\Delta E_{\text {eff }}=2 \hbar / T \simeq 22 \mu \mathrm{eV}$ energy splitting induced by the effective magnetic field.

At the intermediate power, $P=0.95 \mathrm{~mW}$ (see Figure 3), we observe that the excited polariton wavepacket emits Cherenkov radiation at $t \approx 30$ ps (see ref 27 ) and further evolves into a soliton doublet at $t \approx 50 \mathrm{ps}$. At this pump power the polarization dynamics exhibit a drastic change. Formation of a soliton doublet $(t \approx 50 \mathrm{ps})$ is accompanied by the establishment of two almost orthogonally polarized spatially separated polarization domains associated with each of the soliton spatial components. The domain formation is most pronounced in the $S_{1}^{*}$ component $(\mathrm{H}-\mathrm{V}$ linear polarization basis). Figure $3 \mathrm{~d}$ and e show the evolution of the normalized Stokes vectors on the Poincare surface, with one soliton of the doublet in each panel. The Stokes vectors are taken at the positions depicted by the dashed black lines (labeled as "trace 1" and "trace 2") in Figure $3 \mathrm{a}$, each going though the components of the soliton doublet. For trace 1 very fast precession of the soliton Stokes vector around the "south" pole of the Poincare sphere is observed during the first $50 \mathrm{ps}$ (bright red trace in Figure 3d) with a period of $T \simeq 10 \mathrm{ps,} \mathrm{corresponding} \mathrm{to} \mathrm{an} \mathrm{out-of-plane} \mathrm{increased}$ effective magnetic field due to the spin-dependent polariton nonlinearity which induces an energy splitting $\Delta E_{\text {eff }} \simeq 130 \mu \mathrm{eV}$. As the polariton density and hence nonlinearity are reduced at times $t>50$ ps the precession occurs at slower speed with a period of $\sim 100-125$ ps. In this time range the Stokes vectors taken at positions of trace 1 and trace 2 precess in the south and north Poincare hemispheres, respectively, remaining almost orthogonal.

At the highest pump power, $P=3.1 \mathrm{~mW}$ (see Figure 4 ), a further onset of cascaded polariton-polariton scattering leads to the occupation of states on the lower polariton branch at energies below the pump energy in a process resembling the optical continuum generation as observed and discussed in ref 27 for the same MC wire polariton system. In such a process polariton relaxation results in a significant occupation of the states described by positive effective mass, ${ }^{27}$ this results in both coexisting solitons and a spreading dispersive polariton wavepacket with time. At such high polariton density the nonlinearity results in more complex spatiotemporal polarization dynamics, which is shown in Figure 4. The domains with negative and positive circular polarization degree remain for the first $\sim 50-70$ ps as seen for the $S_{3}^{*}$ component in Figure 4c. However, at later times when the nonlinearity becomes weaker, the wavepacket breaks into a set of domains with different polarizations, the Stokes vectors of which evolve in time in a complex manner as shown in Figure $4 d$ and e. This temporal evolution originates from the time- and space-dependent out-ofplane effective magnetic field due to the spin-dependent polariton nonlinearity. 

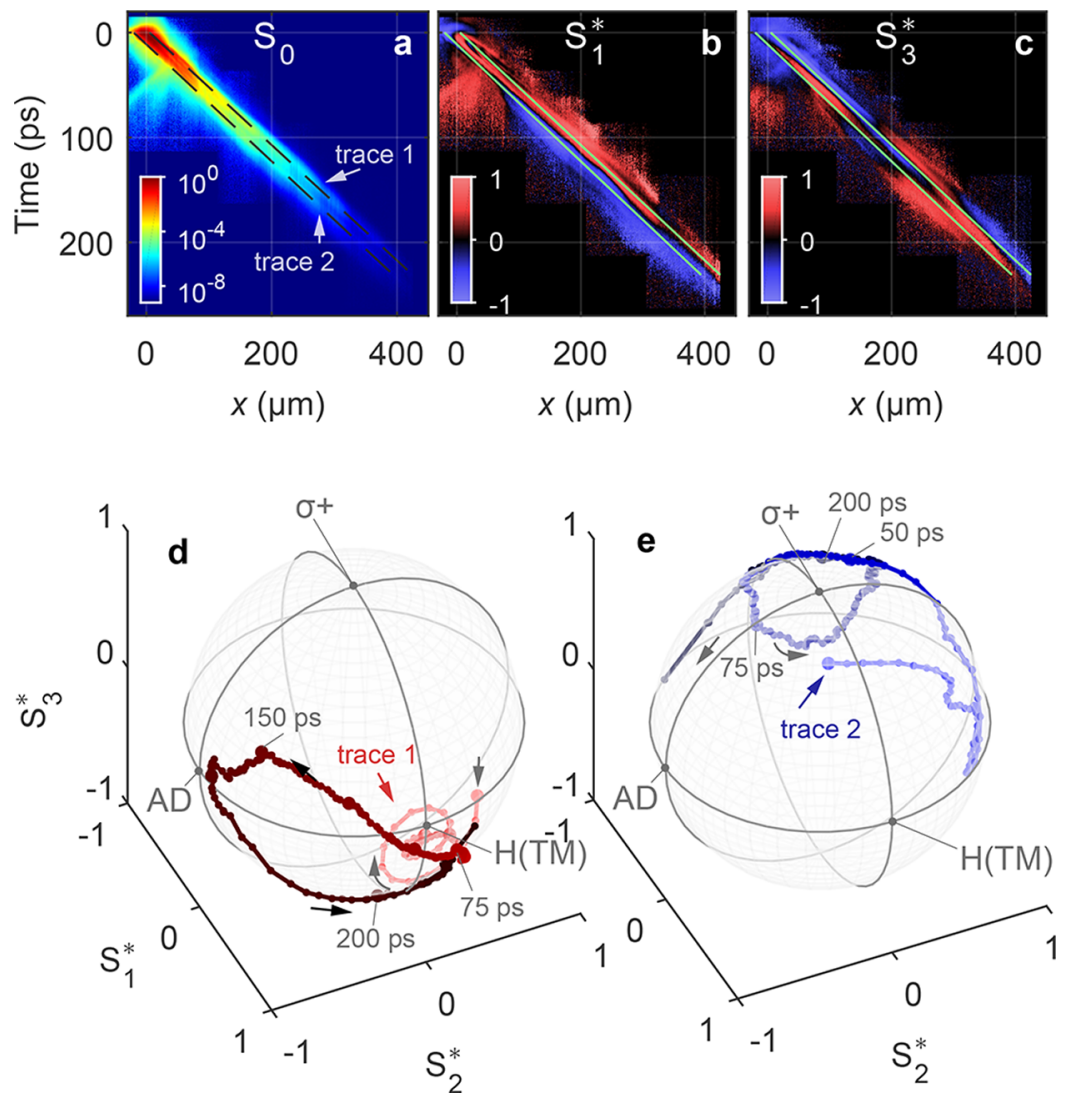

Figure 3. $P=0.95 \mathrm{~mW}$. (a) Total emission intensity $\left(S_{0}\right.$ Stokes component) of a soliton doublet as a function of coordinate $x$ and time. (b, c) Degree of polarization of the emission in linear $\left(S_{1}^{*}\right)$ and circular $\left(S_{3}^{*}\right)$ polarization bases. $(d, e)$ Experimental evolution of the tip of the polariton Stokes vector on the surface of the Poincaré sphere as a function of time measured at the spatial points of the soliton profiles shown by the black dashed/solid green lines in panels $\mathrm{a}, \mathrm{b}$, and $\mathrm{c}$ for traces 1 and 2 , respectively, in panels $\mathrm{d}$ and e. The dimmer (brighter) traces correspond to the evolution on the Stokes vector on the back (front) surface of the sphere. The Stokes vectors are normalized to unity. Data in a-c are taken at $y=0 \pm 0.5 \mu \mathrm{m}$.

\section{MODEL}

In order to describe the polarization dynamics of the nonlinear polariton wavepacket and understand the physical mechanisms responsible for the observed behavior, we work with the coupled spinor macroscopic cavity photon field $\boldsymbol{\psi}=\left(\psi_{+}, \psi_{-}\right)^{T}$ and exciton wave function $\chi=\left(\chi_{+}, \chi_{-}\right)^{T}$ written in the circular polarization basis. ${ }^{34}$ The TE-TM splitting of the photon modes is described by the operator $\Sigma_{\mathrm{k}, \pm}=\beta\left(k_{x} \pm i k_{y}\right)^{2}$ written in reciprocal space ${ }^{35,36}$ and characterized by a splitting constant $\beta$.

Propagation distance of polaritons, as well as the spatial scales of the observed effects, significantly exceed the width of the channel. This allows us to take advantage of the 1D nature of the microwire in order to simplify the formalism. Let us first consider an infinite rectangular potential well width $w_{y}$ where we assume the photon wave function to be in the form $\psi_{ \pm}(t, \mathbf{r})=\sqrt{2 / w_{y}} \cos \left(\pi y / w_{y}\right) \exp \left(-i \varepsilon_{y} t / \hbar\right) \psi_{ \pm}(t, x)$. The set of coupled equations for the $1 \mathrm{D}$ polariton envelope $\psi_{ \pm}(t, x)$ propagating in the $x$-direction can be written

$$
\begin{aligned}
i \hbar \frac{\partial \psi_{ \pm}}{\partial t} & =\left[-\frac{\hbar^{2}}{2 m} \frac{\partial^{2}}{\partial x^{2}}+\Delta-i \frac{\hbar \Gamma}{2}\right] \psi_{ \pm}+\left(\Sigma_{x} \mp i \delta\right) \psi_{\mp} \\
& +\frac{\hbar \Omega}{2} \chi_{ \pm}+E_{ \pm} \mathrm{e}^{-i\left(\omega_{\mathrm{p}} t-k_{\mathrm{p}} x-i x^{2} / 2 w_{x}^{2}-i t^{2} / 2 w_{\mathrm{t}}^{2}\right)}
\end{aligned}
$$

$$
i \hbar \frac{\partial \chi_{ \pm}}{\partial t}=\left[\alpha_{1}\left|\chi_{ \pm}\right|^{2}+\alpha_{2}\left|\chi_{\mp}\right|^{2}-i \frac{\hbar \Gamma_{\chi}}{2}\right] \chi+\frac{\hbar \Omega}{2} \psi_{ \pm}
$$

Excitons possess a much larger effective mass than cavity photons, and their dispersion can be safely approximated as flat compared to the photon dispersion. Here, $m$ is the effective mass of cavity photons; $\Delta$ is the exciton-photon detuning; $\Gamma$ is cavity photon decay rates corresponding to photons leaking from the cavity; $\Gamma_{\chi}$ is the exciton decay rate corresponding to nonradiative dephasing processes; $\Sigma_{x}=-\beta\left(\partial_{x}^{2}+\pi^{2} / w_{y}^{2}\right)$ is the real-space TETM operator along the microwire; $\delta$ describes a fixed splitting in the diagonal polarizations that arises from the optical and electronic anisotropy in a microcavity; ${ }^{32,33}$ and $\hbar \Omega$ is the Rabi splitting giving rise to the exciton-polariton eigenmodes of the system. The parameters $\alpha_{1}$ and $\alpha_{2}$ are the interaction constants in the triplet configuration (parallel spins) and in the singlet configuration (opposite spins), respectively. The last term in eq 4 describes the resonant optical pumping of the lower branch polaritons where $E_{ \pm}$describes the pump pulse amplitude and phase; $w_{x}$ and $w_{\mathrm{t}}$ are the spatial and temporal pulse width, respectively; $\hbar \omega_{\mathrm{p}}$ is the pump energy; and $k_{\mathrm{p}}$ is the pulse wavevector along the wire.

It is instructive to describe the polarization effects in the microwire in terms of an effective magnetic field which acts on the Stokes vector. ${ }^{37}$ The magnetic field vector can be written, in units of energy, as $\boldsymbol{\Omega}=\left(\Omega_{x}, \Omega_{y}, \Omega_{z}\right)$ where $\Omega_{x}=\beta\left(k_{x}^{2}-\pi^{2} / w_{y}^{2}\right)$, $\Omega_{y}=\delta$, and $\Omega_{z}=\left(\alpha_{1}-\alpha_{2}\right)\left(\left|\chi_{+}\right|^{2}-\left|\chi_{-}\right|^{2}\right)$. The last term is a consequence of the nonlinear interactions between polaritons 

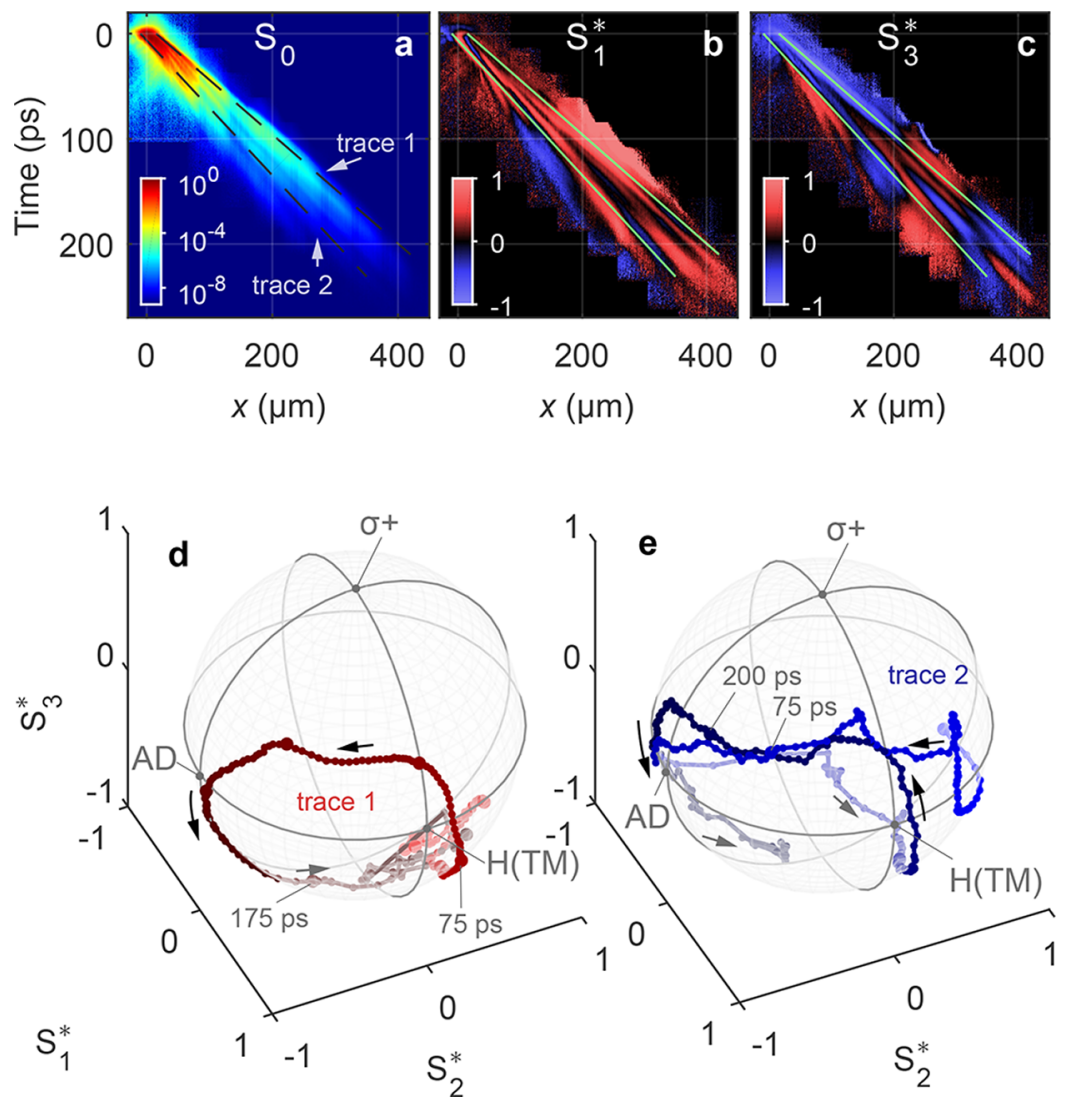

Figure 4. $P=3.1 \mathrm{~mW}$. (a) Total emission intensity $\left(S_{0}\right.$ Stokes component). (b, c) Degree of polarization of the emission in linear $\left(S_{1}^{*}\right)$ and circular $\left(S_{3}^{*}\right)$ polarization bases. (d, e) Experimental evolution of the tip of the polariton Stokes vector on the surface of the Poincare sphere as a function of time for traces 1 and 2, respectively. The Stokes vector was measured at the spatial points of the polariton wavepacket profiles shown by the black dashed (solid green) lines in panel a (b, c). The dimmer (brighter) traces correspond to the evolution on the Stokes vector on the back (front) surface of the sphere. The Stokes vectors are normalized to unity. Data in $a-c$ are taken at $y=0 \pm 0.5 \mu \mathrm{m}$.

giving rise to effective Zeeman splitting when the spin populations are imbalanced.

For modeling, we take the following values of the parameters: $\beta=12 \mu \mathrm{eV} \mu \mathrm{m}^{2} ; \delta_{l}=20 \mu \mathrm{eV} ; \alpha_{1}=2 \mu \mathrm{eV} \mu \mathrm{m} ; \alpha_{2}=-0.1 \alpha_{1}, \Gamma=1 /$ $30 \mathrm{ps}^{-1}, \Gamma_{\chi}=2 \Gamma ; m=5 \times 10^{-5} m_{0}$, where $m_{0}$ is the free electron mass; $\Delta=-2 \mathrm{meV} ; \hbar \Omega=4.12 \mathrm{meV} ; \hbar \omega_{\mathrm{p}}=-1.3 \mathrm{meV} ; k_{\mathrm{p}}=2.2$ $\mu \mathrm{m}^{-1} ; w_{x}$ and $w_{\mathrm{t}}$ were set to have fwhms of $20 \mu \mathrm{m}$ and $5 \mathrm{ps}$, respectively. The initial polarization of the resonant laser is chosen to fit the experimental results: $E_{+}=E_{0}$ and $E_{-}=2.65 e^{i \phi} E_{0}$ where $\phi=1.9$ and $E_{0}$ denotes the overall excitation amplitude of the beam.

\section{SIMULATIONS}

The polarization dynamics of the resonantly excited polariton pulse are shown in Figure $5 \mathrm{a}-\mathrm{c}$ at low excitation power where $\Omega_{z} \ll \Omega_{x}, \Omega_{y}$. The total intensity distribution in Figure 5a already demonstrates the soliton behavior as in the experiment (Figure 2a). Both the linear (Figure 5b) and circular (Figure 5c) polarization components experience harmonic oscillations with frequency $\Omega / \hbar=\sqrt{\beta\left(k_{\mathrm{p}}^{2}-\pi^{2} / w_{y}^{2}\right)+\delta^{2}} / \hbar$ and shifted in phase relative to each other in line with the experimental observations (Figure $2 b, c$ ). We note that in the weak pulse regime the effect of TE-TM splitting does not account for the appearance of oscillations in the linear polarization $S_{1}^{*}$ component since the effective magnetic field is oriented in the $x$-direction. The oscillations of $S_{1}^{*}$ emerge when the additional splitting $\delta$ between the diagonal polarizations is accounted for in the microwire system.

Figure $5 \mathrm{~d}-\mathrm{i}$ show simulation of the nonlinear polarization dynamics of the polariton pulse for intermediate and high excitation powers. Both the intensity (Figure $5 \mathrm{~d}, \mathrm{~g}$ ) and the $S_{1}^{*}$ and $S_{3}^{*}$ polarization (Figure $5 \mathrm{e}, \mathrm{h}$ and $\mathrm{f}, \mathrm{i}$, respectively) effects observed experimentally in Figure $3 b, c$ and Figure $4 b, c$ are reproduced in the modeling. The polariton pulse transforms to a soliton doublet at early stages of propagation as in the experiment (Figure 3a) and is characterized by long streaks of constant $S_{1}^{*}$ polarization as shown in Figure 5e,h, again as in the experiment (see Figure $3 \mathrm{~b}$ ). The reason for this effect is that the $\Omega_{z}$ component decreases as the polariton intensity decays, becoming small or comparable to the in-plane magnetic field $\left(\Omega_{x}, \Omega_{y}\right)$ around $\sim 50$ ps. Consequently, the rotation of the $S_{1}^{*}$ Stokes vector component halts and long streaks are formed in the microwire.

At the low excitation power in Figure $5 \mathrm{a}-\mathrm{c}$ the effective, interaction-induced, $z$-magnetic field has its maximum absolute value of $\Omega_{z} \approx 0.01 \mathrm{meV}$ at around $5 \mathrm{ps}$ after the pulse. The field is small and the polarization precession is mainly governed by splitting between linearly polarized components. Increasing the excitation power, the $\Omega_{z}$ component becomes significant, leading to fast polarization changes. For intermediate pulse energies (Figure $5 \mathrm{~d}-\mathrm{f}$ ) its value is $\Omega_{z} \approx 0.45$ and $0.02 \mathrm{meV}$ at $t \approx$ 20 and $50 \mathrm{ps}$ after the pulse, respectively. For large pulse energies (Figure $5 \mathrm{~g}-\mathrm{i}$ ) its value is $\Omega_{z} \approx 1.6$ and $0.04 \mathrm{meV}$ at $t \approx 10$ and 50 ps after the pulse, respectively. 

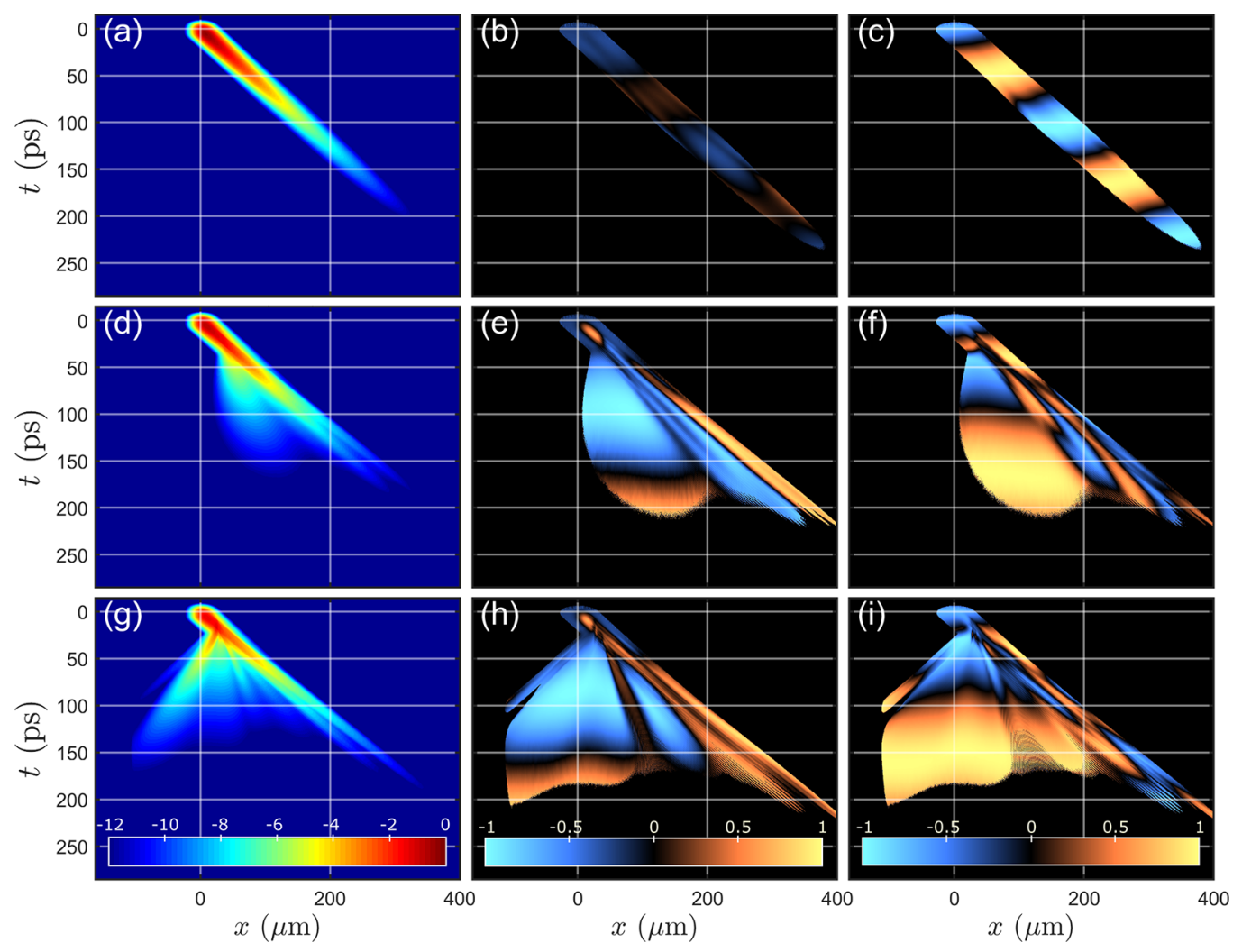

Figure 5. (a, d, g) Total intensity $S_{0}$ (logarithmic scale) and degrees of linear, $S_{1}^{*} \mathrm{~b}, \mathrm{e}, \mathrm{h}$ ), and circular, $S_{3}^{*}(\mathrm{c}, \mathrm{f}, \mathrm{i})$ polarizations. Here $E_{0}=\{0.4,1.5,2.0\}$ $\mathrm{meV} \mu \mathrm{m}^{-1 / 2}$ respectively for top, middle, and bottom panel rows.

Cherenkov radiation is also observed before the pulse separation (Figure 5d,g). At the highest power complex spatiotemporal dynamics of the polarization Stokes components (Figure $5 \mathrm{~h}, \mathrm{i}$ ) resemble those observed in the experiment (Figure $4 b, c)$

We finally point out that although the polariton nonlinearity plays a crucial role in the spin domain formation, the formation of solitons in the system is only weakly influenced by the presence of effective magnetic fields from various polarization splitting mechanisms. We find that approximately the same spatial patterns of solitons appear in the intensity of scalar wave functions and the total intensity of the spinor wave functions considered here (see Section B in the SI). Thus, the initial formation of the solitons at the pump spot (e.g., the pattern of total intensity summed over all polarizations, which leads to nondispersive propagation) is dominantly a scalar effect. Separately, the nonlinear pseudomagnetic field leads to the formation of the polarization domain pattern on top of the solitonic total-intensity pattern.

\section{CONCLUSION}

We have observed nonlinear spin dynamics of polariton wavepackets in a microcavity wire. At low excitation just above the threshold of soliton formation the polarization precession during the propagation is caused by an effective magnetic field due to splittings between linearly polarized polariton components. With increasing polariton density an extra pump-induced out of plane magnetic field due to spin imbalance in the initial polariton wavepacket results in formation of spatially separated polarization domains. While soliton formation is largely a scalar effect (not dependent on polarization), it likely helps to preserve spin domains over the propagation distance due to the nonspreading nature of solitons; hence polarization domains experience little dispersion.

Polariton wires considered in this work can represent building blocks for future polaritonic devices, such as polariton-based spin transistors ${ }^{38,39}$ or soliton-based logic gates. ${ }^{39}$ In the context of the recent demonstration of bright temporal conservative solitons in such a system ${ }^{27}$ polarization domains may be used for the nonbinary information encoding and transfer. This observation also opens a possibility for further fundamental studies of the system, such as description of domain wall formation mechanisms. It would be also interesting to investigate polarization dynamics of bright solitons in high velocity thick waveguide $(>1 \mu \mathrm{m})$ systems, ${ }^{40}$ where the splitting between linearly polarized components is comparable to polariton blue-shifts. Recently, waveguide polaritons have been also reported in a screening-resistant GaN system, ${ }^{41}$ which may potentially work at $300 \mathrm{~K}$, paving the way toward polaritonic device applications.

\section{ASSOCIATED CONTENT}

\section{Supporting Information}

The Supporting Information is available free of charge on the ACS Publications website at DOI: 10.1021/acsphotonics.8b01410.

(1) Numerical simulations demonstrating more clearly the effects of the polariton-polariton interaction induced out-of-plane effective magnetic field on the wavepacket; (2) comparison of scalar and spinor polariton theory on soliton formation; (3) higher resolution experimental images (PDF) 


\section{AUTHOR INFORMATION}

\section{Corresponding Author}

*E-mail: d.krizhanovskii@sheffield.ac.uk.

\section{ORCID $\odot$}

Helgi Sigurdsson: 0000-0002-4156-4414

\section{Notes}

The authors declare no competing financial interest.

\section{ACKNOWLEDGMENTS}

L.E.T.R. acknowledges support from CONACYT, the Mexican Council for Sciences. H.S. and I.A.S. acknowledge support by the Research Fund of the University of Iceland, The Icelandic Research Fund, Grant No. 163082-051. This work was supported by megagrant 14.Y26.31.0015 of the Ministry of Education and Science of the Russian Federation. I.A.S. acknowledges support from Goszadanie No. 3.2614.2017/4.6. M.S.S. and D.N.K. acknowledge the support from UK EPSRC grant EP/N031776/1. A.V.K. acknowledges the St. Petersburg State University for research grant 11.34.2.2012 and the financial support from the Russian Foundation for Basic Research (Project No. 15-52-12018) and Deutsche Forschungsgemeinschaft (DFG) in the framework of International Collaborative Research Center TRR 160.

\section{REFERENCES}

(1) Stenger, J.; Inouye, S.; Stamper-Kurn, D. M.; Miesner, H.-J.; Chikkatur, A. P.; Ketterle, W. Spin domains in ground-state BoseEinstein condensates. Nature 1998, 396, 345.

(2) Sheppard, A. P.; Haelterman, M. Polarization-domain solitary waves of circular symmetry in Kerr media. Opt. Lett. 1994, 19, 859861.

(3) Sánchez-Morcillo, V. J.; Espinosa, V.; Pérez-Arjona, I.; Silva, F.; de Valcárcel, G. J.; Roldán, E. Domain wall dynamics in an optical Kerr cavity. Phys. Rev. E 2005, 71, 066209.

(4) Berkhoer, A. L.; Zakharov, V. E. Self Excitation of Waves with Different Polarizations in Nonlinear Media. Soviet Journal of Experimental and Theoretical Physics 1970, 31, 486.

(5) Kivshar, Y. S.; Agrawal, G. P. Optical Solitonsanytext, 1st ed.; Academic Press, 2003.

(6) Akhmediev, N., Ankiewicz, A., Eds. Dissipative Solitons, 1st ed.; Lect. Notes Phys.; Springer-Verlag: Berlin Heidelberg, 2005; Vol. 661; p 448.

(7) Skryabin, D. V.; Gorbach, A. V. Colloquium: Looking at a soliton through the prism of optical supercontinuum. Rev. Mod. Phys. 2010, 82, 1287-1299.

(8) Gilles, M.; Bony, P. Y.; Garnier, J.; Picozzi, A.; Guasoni, M.; Fatome, J. Polarization domain walls in optical fibres as topological bits for data transmission. Nat. Photonics 2017, 11, 102-107.

(9) Marconi, M.; Javaloyes, J.; Barland, S.; Balle, S.; Giudici, M. Vectorial dissipative solitons in vertical-cavity surface-emitting lasers with delays. Nat. Photonics 2015, 9, 450.

(10) Gippius, N. A.; Shelykh, I. A.; Solnyshkov, D. D.; Gavrilov, S. S.; Rubo, Y. G.; Kavokin, A. V.; Tikhodeev, S. G.; Malpuech, G. Polarization Multistability of Cavity Polaritons. Phys. Rev. Lett. 2007, 98, 236401.

(11) Walker, P. M.; Tinkler, L.; Skryabin, D. V.; Yulin, A.; Royall, B.; Farrer, I.; Ritchie, D. A.; Skolnick, M. S.; Krizhanovskii, D. N. Ultra-lowpower hybrid light-matter solitons. Nat. Commun. 2015, 6, 8317.

(12) Proukakis, N. P.; Snoke, D. W.; Littlewood, P. B., Eds. Universal Themes of Bose-Einstein Condensation; Cambridge University Press, 2017.

(13) Sich, M.; Skryabin, D. V.; Krizhanovskii, D. N. Physique des solitons avec des polaritons excitoniques semiconducteurs dans des systèmes confinés. C. R. Phys. 2016, 17, 908-919.
(14) Shelykh, I. A.; Kavokin, A. V.; Rubo, Y. G.; Liew, T. C. H.; Malpuech, G. Polariton polarization-sensitive phenomena in planar semiconductor microcavities. Semicond. Sci. Technol. 2010, 25, 013001.

(15) Shelykh, I.; Kavokin, K. V.; Kavokin, A. V.; Malpuech, G.; Bigenwald, P.; Deng, H.; Weihs, G.; Yamamoto, Y. Semiconductor microcavity as a spin-dependent optoelectronic device. Phys. Rev. B: Condens. Matter Mater. Phys. 2004, 70, 035320.

(16) Ciuti, C.; Savona, V.; Piermarocchi, C.; Quattropani, A.; Schwendimann, P. Role of the exchange of carriers in elastic excitonexciton scattering in quantum wells. Phys. Rev. B: Condens. Matter Mater. Phys. 1998, 58, 7926-7933.

(17) Wouters, M. Resonant polariton-polariton scattering in semiconductor microcavities. Phys. Rev. B: Condens. Matter Mater. Phys. 2007, 76, 045319.

(18) Vladimirova, M.; Cronenberger, S.; Scalbert, D.; Kavokin, K. V.; Miard, A.; Lemaître, A.; Bloch, J.; Solnyshkov, D.; Malpuech, G.; Kavokin, A. V. Polariton-polariton interaction constants in microcavities. Phys. Rev. B: Condens. Matter Mater. Phys. 2010, 82, 075301.

(19) Amo, A.; Liew, T. C. H.; Adrados, C.; Houdré, R.; Giacobino, E.; Kavokin, A. V.; Bramati, A. Exciton-polariton spin switches. Nat. Photonics 2010, 4, 361-366.

(20) Gao, T.; Antón, C.; Liew, T. C. H.; Martín, M. D.; Hatzopoulos, Z.; Viña, L.; Eldridge, P. S.; Savvidis, P. G. Spin selective filtering of polariton condensate flow. Appl. Phys. Lett. 2015, 107, 011106.

(21) Sich, M.; Fras, F.; Chana, J. K.; Skolnick, M. S.; Krizhanovskii, D. N.; Gorbach, A. V.; Hartley, R.; Skryabin, D. V.; Gavrilov, S. S.; CerdaMéndez, E. A.; Biermann, K.; Hey, R.; Santos, P. V. Effects of SpinDependent Interactions on Polarization of Bright Polariton Solitons. Phys. Rev. Lett. 2014, 112, 046403.

(22) Ohadi, H.; Ramsay, A. J.; Sigurdsson, H.; del Valle-Inclan Redondo, Y.; Tsintzos, S. I.; Hatzopoulos, Z.; Liew, T. C. H.; Shelykh, I. A.; Rubo, Y. G.; Savvidis, P. G.; Baumberg, J. J. Spin Order and Phase Transitions in Chains of Polariton Condensates. Phys. Rev. Lett. 2017, $119,067401$.

(23) Sigurdsson, H.; Ramsay, A. J.; Ohadi, H.; Rubo, Y. G.; Liew, T. C. H.; Baumberg, J. J.; Shelykh, I. A. Driven-dissipative spin chain model based on exciton-polariton condensates. Phys. Rev. B: Condens. Matter Mater. Phys. 2017, 96, 155403.

(24) Hivet, R.; Flayac, H.; Solnyshkov, D. D.; Tanese, D.; Boulier, T.; Andreoli, D.; Giacobino, E.; Bloch, J.; Bramati, A.; Malpuech, G.; Amo, A. Half-solitons in a polariton quantum fluid behave like magnetic monopoles. Nat. Phys. 2012, 8, 724-728.

(25) Rubo, Y. G. Half Vortices in Exciton Polariton Condensates. Phys. Rev. Lett. 2007, 99, 106401.

(26) Dominici, L.; Carretero-González, R.; Gianfrate, A.; CuevasMaraver, J.; Rodrigues, A. S.; Frantzeskakis, D. J.; Lerario, G.; Ballarini, D.; De Giorgi, M.; Gigli, G.; Kevrekidis, P. G.; Sanvitto, D. Interactions and scattering of quantum vortices in a polariton fluid. Nat. Commun. 2018, 9, 1467.

(27) Skryabin, D. V.; Kartashov, Y. V.; Egorov, O. A.; Sich, M.; Chana, J. K.; Tapia Rodriguez, L. E.; Walker, P. M.; Clarke, E.; Royall, B.; Skolnick, M. S.; Krizhanovskii, D. N. Backward Cherenkov radiation emitted by polariton solitons in a microcavity wire. Nat. Commun. 2017, 8,1554 .

(28) Tinkler, L.; Walker, P. M.; Clarke, E.; Krizhanovskii, D. N.; Bastiman, F.; Durska, M.; Skolnick, M. S. Design and characterization of high optical quality InGaAs/GaAs/AlGaAs-based polariton microcavities. Appl. Phys. Lett. 2015, 106, 021109.

(29) Sich, M.; Krizhanovskii, D. N.; Skolnick, M. S.; Gorbach, A. V.; Hartley, R.; Skryabin, D. V.; Cerda-Méndez, E. A.; Biermann, K.; Hey, R.; Santos, P. V. Observation of bright polariton solitons in a semiconductor microcavity. Nat. Photonics 2012, 6, 50-55.

(30) Kavokin, K. V.; Shelykh, I. A.; Kavokin, A. V.; Malpuech, G.; Bigenwald, P. Quantum Theory of Spin Dynamics of ExcitonPolaritons in Microcavities. Phys. Rev. Lett. 2004, 92, 017401.

(31) Langbein, W.; Shelykh, I.; Solnyshkov, D.; Malpuech, G.; Rubo, Y.; Kavokin, A. Polarization beats in ballistic propagation of excitonpolaritons in microcavities. Phys. Rev. B: Condens. Matter Mater. Phys. 2007, 75, 075323. 
(32) Amo, A.; Liew, T. C. H.; Adrados, C.; Giacobino, E.; Kavokin, A. V.; Bramati, A. Anisotropic optical spin Hall effect in semiconductor microcavities. Phys. Rev. B: Condens. Matter Mater. Phys. 2009, 80, 165325.

(33) Glazov, M. M.; Golub, L. E. Spin and transport effects in quantum microcavities with polarization splitting. Phys. Rev. B: Condens. Matter Mater. Phys. 2010, 82, 085315.

(34) Carusotto, I.; Ciuti, C. Probing Microcavity Polariton Superfluidity through Resonant Rayleigh Scattering. Phys. Rev. Lett. 2004, 93, 166401.

(35) Panzarini, G.; Andreani, L. C.; Armitage, A.; Baxter, D.; Skolnick, M. S.; Astratov, V. N.; Roberts, J. S.; Kavokin, A. V.; Vladimirova, M. R.; Kaliteevski, M. A. Exciton-light coupling in single and coupled semiconductor microcavities: Polariton dispersion and polarization splitting. Phys. Rev. B: Condens. Matter Mater. Phys. 1999, 59, 50825089.

(36) Kavokin, A.; Malpuech, G.; Glazov, M. Optical Spin Hall Effect. Phys. Rev. Lett. 2005, 95, 136601.

(37) Kavokin, K. V.; Shelykh, I. A.; Kavokin, A. V.; Malpuech, G.; Bigenwald, P. Quantum Theory of Spin Dynamics of ExcitonPolaritons in Microcavities. Phys. Rev. Lett. 2004, 92, 017401.

(38) Solnyshkov, D. D.; Johne, R.; Shelykh, I. A.; Malpuech, G. Chaotic Josephson oscillations of exciton-polaritons and their applications. Phys. Rev. B: Condens. Matter Mater. Phys. 2009, 80, 235303.

(39) Cancellieri, E.; Chana, J. K.; Sich, M.; Krizhanovskii, D. N.; Skolnick, M. S.; Whittaker, D. M. Logic gates with bright dissipative polariton solitons in Bragg cavity systems. Phys. Rev. B: Condens. Matter Mater. Phys. 2015, 92, 1-5.

(40) Shapochkin, P. Y.; Lozhkin, M. S.; Solovev, I. A.; Lozhkina, O. A.; Efimov, Y. P.; Eliseev, S. A.; Lovcjus, V. A.; Kozlov, G. G.; Pervishko, A. A.; Krizhanovskii, D. N.; Walker, P. M.; Shelykh, I. A.; Skolnick, M. S.; Kapitonov, Y. V. Polarization-resolved strong light-matter coupling in planar GaAs/AlGaAs waveguides. Opt. Lett. 2018, 43, 4526.

(41) Ciers, J.; Roch, J.; Carlin, J.-F.; Jacopin, G.; Butté, R.; Grandjean, N. Propagating Polaritons in III-Nitride Slab Waveguides. Phys. Rev. Appl. 2017, 7, 034019. 